\title{
Fractura de cadera en el paciente mayor de 90 años: nuestro nuevo desafío
}

\section{Hip Fracture in the Extremely Elderly: Our New Dilemma}

\author{
Camila Azócar Sanhueza ${ }^{1}$ Javier González Almonacid ${ }^{2}$ José Ignacio Quezada Morales ${ }^{2}$ \\ Khader Sabat Villazón ${ }^{2}$ Roberto Valiente Valenzuela ${ }^{3}$ Christian Hübner Hoffman ${ }^{4}$ \\ Rodrigo Liendo Verdugo ${ }^{5}$
}

${ }^{1}$ Departamento de Ortopedia y Traumatología Universidad de Chile,
Santiago, Chile
2 Residentes programa de formación de especialista de Ortopedia y
Traumatología de la Universidad de Chile
${ }^{3}$ Servicio de Ortopediay Traumatología, Clínica Santa María, Santiago, Chile
${ }^{4}$ Servicio de Traumatología Hospital de Urgencia Asistencia Pública,
Santiago, Chile
${ }^{5}$ Departamento de Ortopedia y Traumatología Universidad Católica
de Chile

Address for correspondence Camila Azócar Sanhueza, Santa María 0500, Providencia, Santiago, Chile (e-mail: camiazocars@gmail.com).

Rev Chil Ortop Traumatol 2018;59:65-71.

\section{Resumen}

Palabras Clave

- fractura de cadera

- paciente añoso

- nonagenario
Introducción El envejecimiento mundial de la población ha resultado en un aumento de las fracturas de cadera y sobre todo en pacientes cada vez más añosos. El objetivo es buscar la mortalidad al año y factores asociados en nuestra realidad nacional.

Materiales y Métodos Estudio retrospectivo observacional de fichas clínicas e imagenología de pacientes mayores de 90 años operados por fractura de cadera en la capital de nuestro país. Se presentan dos grupos según su sistema previsional, pacientes pertenecientes al sistema de salud público (SSPu) y pertenecientes a un sistema de salud privado (SSPr). Se realiza análisis estadístico univariado y multivariado con test de Chi ${ }^{2}$, Mann-Whitney y regresión logística.

Resultados 129 pacientes mayores de 90 años operados por fractura de cadera (85 pertenecientes al SSPu y 44 en el SSPr), cumplieron con criterios de inclusión y exclusión en un período de 2 años. El promedio de edad general fue de 93,2 años (SD $2,9)$ sin diferencia entre grupos. El tiempo preoperatorio fue significativamente menor en el SSPr con mediana de 1 día (0-7) y de 6 días (1-40) para el SSPu $(p<0,001)$. La mortalidad general al año fue de $30,2 \%$ siendo de $38,8 \%$ para el SSPu y de $13,6 \%$ para el SSPr con diferencia significativa $(p=0,003)$. No se encontró asociación estadística en la mortalidad al año con las variables edad, género ni tipo de fractura. Se encontró asociación entre la mortalidad al año y menor tiempo preoperatorio $(p<0,001)$. En el análisis multivariado de mortalidad el único factor protector fue pertenecer al SSPr $(p=0,010)$.

Discusión La mortalidad general de nuestros pacientes es similar a lo encontrado en la literatura internacional, siendo el menor tiempo preoperatorio y pertenecer al sistema de salud privado factores asociados a menor mortalidad al año, donde ese received

October 28, 2017

accepted

July 18, 2018

published online

August 13, 2018
DOI https://doi.org/

10.1055/s-0038-1668512. ISSN 0716-4548.
Copyright (e 2018 by Thieme Revinter

Publicações Ltda, Rio de Janeiro, Brazil

\section{License terms}

(1) (1) $\Theta \circledast$ 
último es el único factor asociado en el estudio multivariable. Este estudio aporta a la contingencia nacional en describir cómo se comporta nuestra población tanto en el sistema de salud privado como en el público. Es necesario estudiar el efecto en la mortalidad de otros factores asociados que no fueron evaluados en el presente trabajo.

\begin{abstract}
Introduction The aging of world population has resulted in an increase of hip fractures and especially in extremely elderly patients. The objective is to describe 1year mortality in these patients in our national reality.

Materials and Methods Observational retrospective study of clinical records and imaging of patients over 90 years old who were operated for hip fracture in the capital of our country, belonging one group to private care health system ( $\mathrm{SSPr}$ ) and the other group to the public health system (SSPu). Statistical analysis was performed to type of implant and mortality.

Results 129 patients over 90 years old were operated for hip fracture (85 in the SSPu and 44 in the SSPr) in a period of 2 years. The average age was of 93.2 years (SD 2.9) without statistical difference between groups. Preoperative time was significantly lower in $\mathrm{SSPr}$ group with mean of 1 day (0-7) for the SSPr and 6 days (1-40) for the SSPu $(p<0,001)$. The overall 1-year mortality was $30.2 \% ; 38.8 \%$ in the SSPu and $13.6 \% \%$, which was statistically relevant $(p=0,003)$. No association was found between 1-year mortality and age, sex or fracture type. Statistical association was found between preoperative time and 1-year mortality where patients who have lower preoperative time have lower 1 -year mortality $(p<0,001)$. Association was found between lower 1 -year mortality and belonging to SSPr. This last association was the only one with statistical relevance in the multivariable analysis $(p=0,010)$

Discussion Surgery for hip fractures in extremely elderly patients is an increasing reality in our country. The overall mortality of our patients is similar to that published in the international literature, where belonging to private care health system and having a low

\section{Keywords}

- hip fracture

- extremly elderly

- nonagenarians preoperative time is associated with a lower 1-year mortality in our study. This study contributes to the national contingency in the sense of how our population develops in the private sphere as well as in the public care. It is necessary to study the effect on mortality of other associated factors that were not evaluated in the present study.
\end{abstract}

\section{Introducción}

El envejecimiento de la población a nivel mundial es una realidad que constituye un verdadero dilema a la hora de la toma de decisiones en el ámbito médico. ${ }^{1}$ Entre los años 2000 y 2050, la proporción de habitantes mayores de 60 años se duplicará constituyendo un $22 \%$ de la población mundial y los mayores de 80 años aumentarán casi cuatro veces alcanzando aproximadamente 395 millones de habitantes. ${ }^{1}$

Chile es actualmente el país con mayor esperanza de vida al nacer de América Latina. ${ }^{2}$ Hay aproximadamente 2 millones de personas mayores de 60 años con 417.000 sobre 80 años en nuestro país, lo que constituye el $11 \%$ de la población. ${ }^{3}$

Se sabe que la población anciana es más vulnerable a las caídas donde un 35\% de las personas mayores de 65 años presentan caídas cada año ${ }^{4}$ lo que se traduce en un aumento global de pacientes con fracturas de caderas y sobre todo en una población que cada vez es más añosa. ${ }^{4}$
Estudios previos han demostrado un aumento en la mortalidad asociado a la edad avanzada posterior a la fractura de cadera, ${ }^{5-12}$ donde los mayores de 90 años tienen mayor mortalidad durante la hospitalización y mayor mortalidad al año en comparación con pacientes de menor edad. ${ }^{5}$ Además, la edad avanzada está asociada a menor recuperación funcional, menor capacidad de marcha y mayor frecuencia y severidad de complicaciones postoperatorias. ${ }^{5-12}$

Si bien esta población ha sido ampliamente descrita en la literatura internacional, no existen registros nacionales de mortalidad en ese subgrupo de pacientes. El presente estudio, busca describir la epidemiología de las fracturas de pacientes mayores de 90 años en nuestra realidad nacional, y comparar resultados entre pacientes operados por fracturas de cadera pertenecientes al sistema previsional de salud privado con los del sistema de salud público.

Objetivo primario: Describir la mortalidad al año de pacientes mayores de 90 años operados por fractura de cadera. 
Objetivos secundarios: Describir la epidemiología de los pacientes mayores de 90 años operados por fractura de cadera, comparar resultados de mortalidad entre los distintos sistemas de salud al que pertenecen los pacientes y buscar asociación de mortalidad y tiempo preoperatorio.

\section{Materiales y Métodos}

Se realizó un estudio retrospectivo observacional de fichas y radiografías de pacientes operados por fractura de cadera en dos centros hospitalarios de Santiago de Chile, uno donde los pacientes son pertenecientes al sistema previsional de salud público ( $\mathrm{SSPu}$ ) y otro donde los pacientes pertenecen a un sistema de salud privado (SSPr), entre enero del $2012 \mathrm{y}$ diciembre del 2014. Se obtuvieron datos demográficos de género, edad y tiempo preoperatorio (tiempo transcurrido desde el ingreso del paciente hasta el día de la cirugía) desde las fichas clínicas. Se obtuvo las fechas de defunción a través de datos públicos del Registro Civil de Chile con un mínimo de 1 año de seguimiento. Se realizó un estudio de radiografías pre $\mathrm{y}$ postoperatorias por 3 de los autores quienes independientemente clasificaron las fracturas en extracapsular e intracapsular y en las subclasificaciones de ambas: Garden para las fracturas intracapsulares y Tronzo para las fracturas extracapsulares (el total de radiografías fue dividido entre los observadores y corregidos por el autor principal del estudio). Además, se obtuvieron datos sobre tipos de osteosíntesis y artroplastías de los protocolos operatorios.

Los criterios de inclusión fueron: Pacientes operados por fractura de cadera, cirugías entre enero del 2012 y diciembre del 2014, mayores de 90 años. Los criterios de exclusión fueron: falta de datos demográficos en fichas clínicas, protocolos y ausencia de radiografías en los sistemas de almacenamiento imagenológicos de cada centro.

Para el análisis estadístico se utilizó el test de ShapiroWilk para evaluar la distribución normal de las variables ordinales, el test de $\mathrm{Chi}^{2}$ en variables paramétricas, test de Mann-Whitney para variables no paramétricas y regresión logística para el análisis multivariable.

\section{Resultados}

Se registró un total de 146 pacientes operados por fractura de cadera mayores de 90 años durante el período estudiado de los cuales 129 cumplieron con criterios de inclusión y exclusión. De los 129 paciente incluidos en el estudio 85 $(65,9 \%)$ pertenecían al SSPu y $44(34,1 \%)$ al SSPr.

El promedio de edad general fue de 93,2 años (SD 2,9) siendo de 93,8 años (SD 3,6) para el SSPr y de 92,8 años (SD $2,6)$ para el SSPu. Las medianas respectivas fueron de 92,5 (90-103) y 92 (90-100) años respectivamente para ambos grupos sin diferencia estadística ( $p=0,1995$ test MannWhitney). La demografía de los pacientes se presenta en la - Tabla 1. El género femenino fue predominante en ambos sistemas de salud sin diferencia. Se encontró diferencia entre los dos sistemas de salud en el tipo de fractura siendo las extracapsulares predominantes en el SSPu y las intracapsulares en el $\operatorname{SSPr}(p<0,001)$. No se encontró diferencia al comparar los tipos de fractura según género $(p=0,948)$.

La subclasificación del tipo de fractura se muestra en las - Figs. 1 y 2 y los tipos de implantes utilizados para cada tipo de fractura en los distintos sistemas de salud se muestran en las - Figs. 3 y 4.

El tiempo preoperatorio promedio fue de 6,7 días (SD 7,7) con una mediana de 5 días (0-40) donde la mediana para el SSPr fue de 1 días (0-7) y de 6 días (1-40) para el SSPu $(p<0,001)$. Al considerar como punto de corte las 72 horas, encontramos que el 95,5\% (42/44 pacientes) de los pacientes operados en el SSPr cumplieron ese criterio mientras que eso solo ocurrió en el $12,9 \%$ (11/85 pacientes) de los pertenecientes al SSPu $(p<0,001)$.

La mortalidad general fue de $30,2 \%$ al año encontrándose una mortalidad al año de $13,6 \%$ para el SSPr y de $38,8 \%$ para el SSPu con diferencia significativa $\left(p=0,003\right.$ test $\left.\mathrm{Chi}^{2}\right)$. Se realiza curva de Kaplan-Meyer para graficar sobrevida al año ( - Fig.5).

Al realizar análisis univariable, no se encontró asociación de la mortalidad con la edad ( $p=0,79)$, género $(p=0,222)$ ni tipo de fractura $(p=0,292)$. Si se encontró asociación entre la mortalidad con el tiempo preoperatorio $(p=0,0087)$ donde a mayor tiempo preoperatorio, mayor mortalidad al año.

Se realizó estudio multivariable mediante regresión logística que se muestra en la - Tabla 2. Para realizar el análisis, la edad y el tiempo preoperatorio se consideraron variables categóricas donde para la edad se utilizó como punto de corte la mediana (92 años) y para el tiempo preoperatorio las 72 horas desde el ingreso. Encontramos que el único factor protector de mortalidad es pertenecer al $\operatorname{SSPr}(p=0,010)$.

Tabla 1 Resumen descriptivo de pacientes estudiados separados según sistema de salud

\begin{tabular}{|l|l|l|l|l|l|}
\hline Variable & Total & SSPr & SSPu & Valor-p \\
\hline \multirow{2}{*}{ Edad } & Promedio \pm d.e & $93,2 \pm 2,9$ & $93,8 \pm 3,6$ & $92,8 \pm 2,6$ & $0,1995^{*}$ \\
\cline { 2 - 6 } & Mediana (rango) & $92(90-103)$ & $92,5(90-103)$ & $92(90-100)$ & $0,187^{* *}$ \\
\hline \multirow{2}{*}{ Género n (\%) } & Mujeres & $110(85,3)$ & $35(79,5)$ & $75(88,2)$ & $10(11,8)$ \\
\cline { 2 - 6 } & Hombres & $19(14,7)$ & $9(20,5)$ & $56(65,9)$ & $<0,001^{* *}$ \\
\hline $\begin{array}{l}\text { Tipo de n (\%) } \\
\text { fractura }\end{array}$ & Extracap. & $67(51,9)$ & $11(25)$ & $29(34,1)$ & \\
\cline { 2 - 6 } & Intracap. & $62(48,1)$ & $33(75)$ & & 2 \\
\hline
\end{tabular}

${ }^{*}$ Test de Mann-Whitney, ${ }^{* *}$ Test de Chi. ${ }^{2}$ 

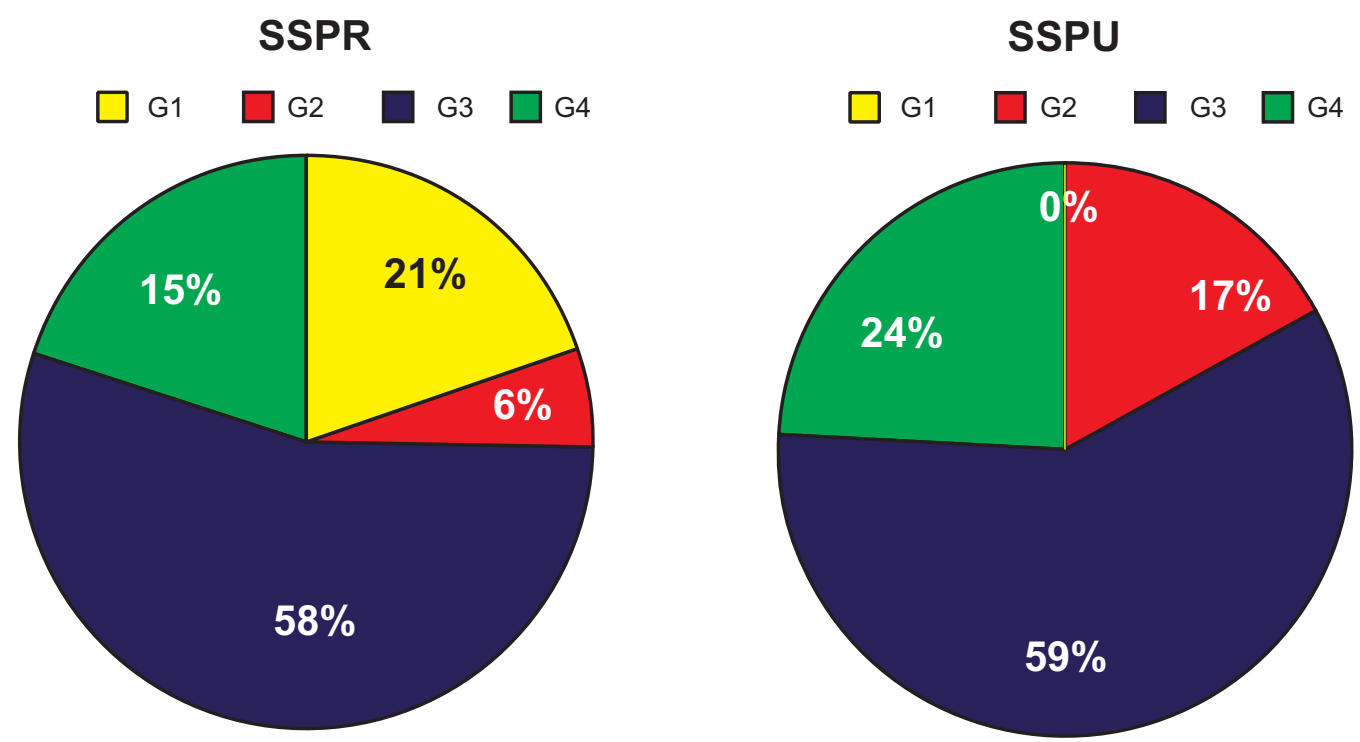

Fig. 1 Clasificación de Garden (G) en Fracturas Intracapsulares Según Sistema de Salud.

\section{SSPR}

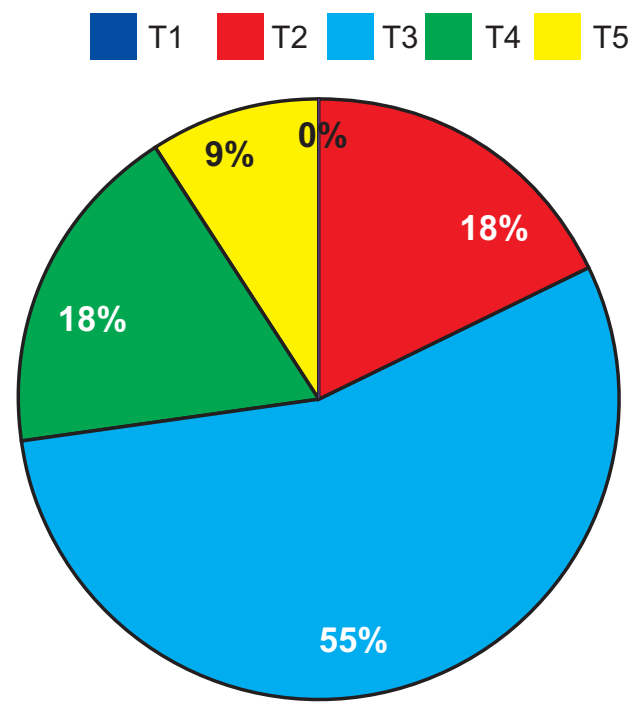

SSPU

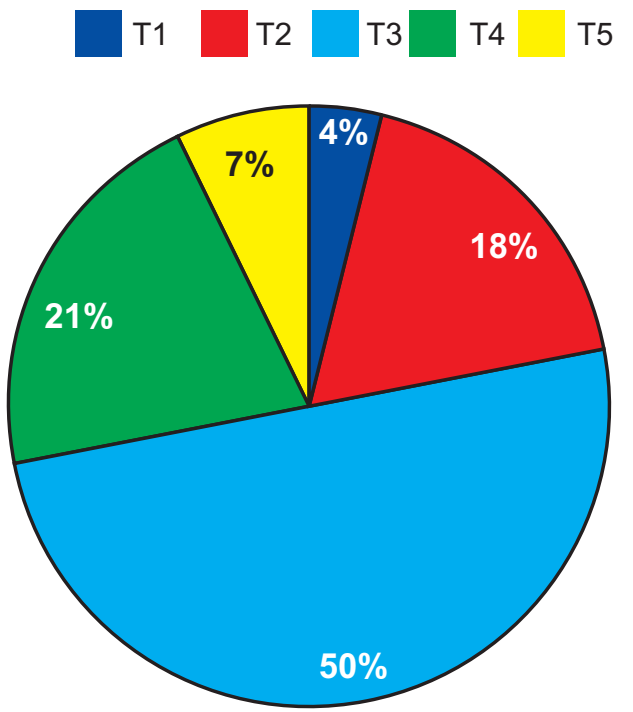

Fig. 2 Clasificación de Tronzo (T) en Fracturas Extracapsulares Según Sistema de Salud.

\section{Discusión}

La fractura de cadera es una patología frecuente y se considera una de las causas más importante de morbimortalidad en el adulto mayor. El envejecimiento de la población nos obliga a enfrentarnos a un creciente número de pacientes nonagenarios con fractura de cadera que deben ser sometidos a cirugía y se convierte en un desafío para el cirujano.

En el presente estudio se describen pacientes mayores de 90 años que fueron sometidos a cirugía por fractura de cadera afiliados a dos sistemas previsionales de salud en nuestro país SSPu y SSPr. El promedio de edad de los pacientes evaluados fue de 93,2 años y se encontró una predominancia significativa de pacientes de género femenino, lo cual se condice con lo publicado en la literatura internacional. ${ }^{7,13,14}$

Al clasificar las fracturas en intracapsulares y extracapsulares, encontramos una diferencia significativa entre ambos centros, siendo más frecuente la fractura intracapsular (75\%) en los pacientes operados en el SSPr mientras que la fractura extracapsular fue más prevalente en pacientes del SSPu (65,9\%). Los estudios publicados con respecto a ese tema son controversiales encontrándose estudios donde predominan las fracturas extracapsulares y otros donde son superadas por las fracturas intracapsulares. Por ejemplo, Gjertsen y col., describe mayor prevalencia de fracturas intracapsulares en la población de Noruega. ${ }^{15} \mathrm{Sin}$ embargo, en la mayoría de los casos, se describe una distribución similar sin diferencias estadísticas. ${ }^{13,16}$ 


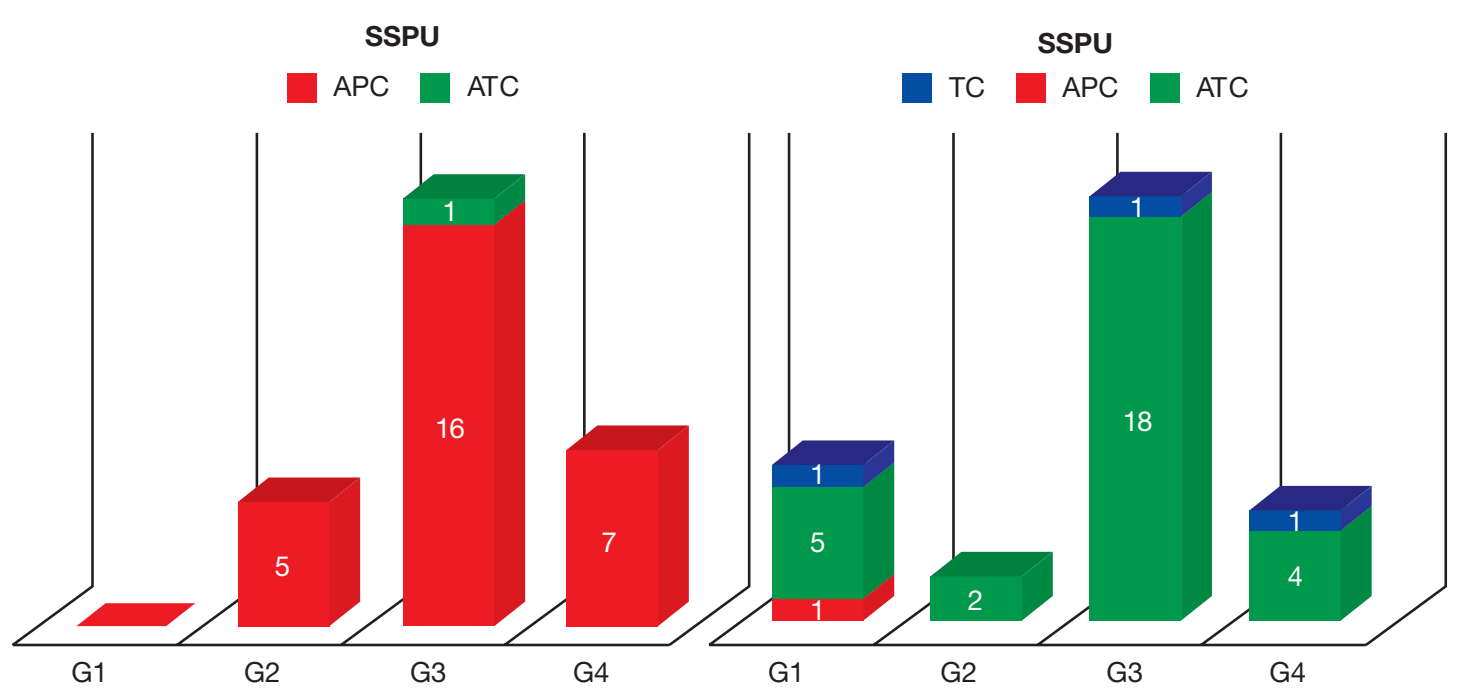

Fig. 3 Tipos de Implante en Fracturas Intracapsulares Según Sistema de Salud. Abreviaciones: APC, artroplastía parcial de cadera; CCM, clavo cefalomedular; DHS, dynamic hip screw; G, garden.

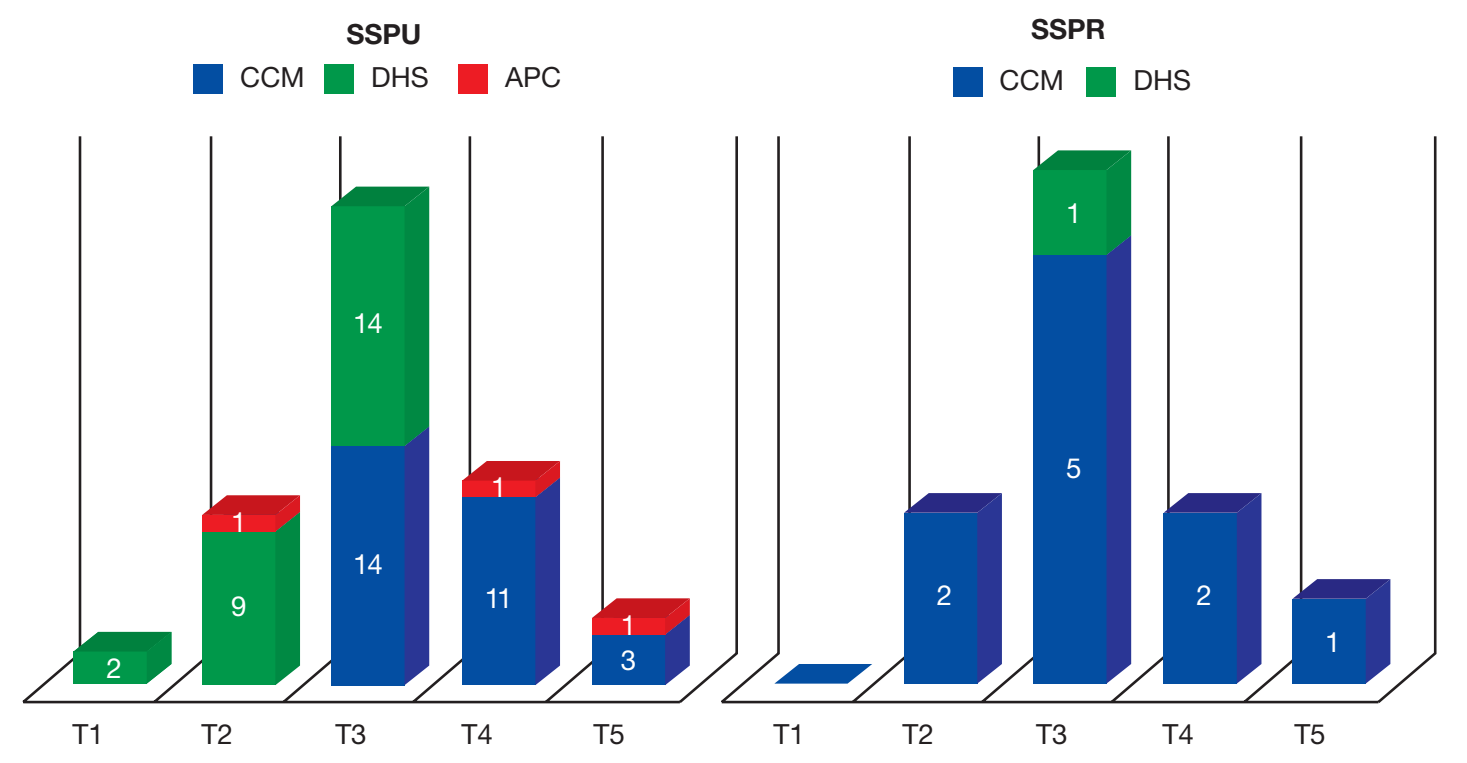

Fig. 4 Tipos de Implante en Fracturas Extracapsulares Según Sistema de Salud. Abreviaciones: APC, artroplastía parcial de cadera; ATC, artroplastía total de cadera; T, tronzo; TC, tornillos canulados.

Con respecto a la subclasificación y tipos de implantes utilizados en los distintos sistemas de salud, se muestran los resultados obtenidos, pero no se pudo realizar un análisis estadístico que permitiese realizar conclusiones puesto que la cantidad de pacientes en cada subgrupo es muy pequeña y eso conlleva a un error estadístico.

El tiempo preoperatorio promedio general fue de 6,7 días (DS 7,6) siendo ese significativamente mayor en los pacientes pertenecientes al SSPu destacando que solo el 12,9\% de los pacientes del SSPu son operados antes de las 72 horas a diferencia del 95,5\% de los pacientes del SSPr. Se utilizó ese punto de corte ya que es el valor utilizado en la literatura para describir cirugía precoz y al cual se le adjudica una menor mortalidad. ${ }^{17}$
La mortalidad general al año encontrada en nuestro estudio es de $30,2 \%$, comparable con lo encontrado en la literatura internacional donde existe gran dispersión de resultados encontrando estudios como el de Lin y col., ${ }^{18}$ que encuentra una mortalidad al año de $17,3 \%$ mientras que de Leur ${ }^{18}$ y col., de $36 \%$ en pacientes nonagenarios. Ese dato es de gran relevancia nacional puesto que no existen publicaciones previas sobre la mortalidad al año en pacientes añosos operados por fractura de cadera en nuestro país.

Nuestro estudio no consideró variables de comorbilidad de los pacientes para el análisis de mortalidad. Eso es una limitación importante para poder identificar factores asociados a mal pronóstico, puesto que en la literatura internacional hay consenso de que esos factores y sobre 




Fig. 5 Curva Kaplan-Meyer sobrevida a 1 año.

todo la clasificación de la academia americana de anestesia (ASA), son factores asociados a mortalidad con significancia estadística. ${ }^{13,19-23}$ Más aun considerando que sobre el 60\% de los pacientes con fractura de cadera tienen comorbilidades asociadas. $^{22}$

Se encontró una asociación estadística entre el menor tiempo preoperatorio y la menor mortalidad al año en el análisis univariable. Ese resultado es similar al encontrado en la literatura donde Simunovic y col., ${ }^{17}$ en su revisión sistemática y metaanálisis, encuentran una menor mortalidad en pacientes operados antes de las 24-72hrs. Sin embargo, ese factor puede estar influenciado por la presencia de comorbilidades que pudiesen tener un papel en el retraso de la cirugía, por lo que se hace necesario realizar estudios que incluyan esta variable para poder discriminar entre factores confundentes.

No encontramos asociación en el análisis univariable de la mortalidad con las variables de género, edad ni tipo de fractura, encontrándose resultados similares en la literatura internacional. ${ }^{24}$

Tabla 2 Análisis Multivariable con Regresión Logística

\begin{tabular}{|l|l|l|l|}
\hline Variable & Coeficiente & $\begin{array}{l}\text { Intervalo } \\
\text { de Confianza }\end{array}$ & $\mathbf{P}>\mathbf{t}$ \\
\hline Pertenecer al SSPr & $-0,378$ & $-0,664-0,093$ & $0,010^{*}$ \\
\hline Género Femenino & $-0,181$ & $-0,408-0,044$ & 0,114 \\
\hline $\begin{array}{l}\text { Tiempo } \\
\text { preoperatorio } \\
\text { menor a 72horas }\end{array}$ & $-0,120$ & $-0,143-0,383$ & 0,368 \\
\hline $\begin{array}{l}\text { Edad menor de } \\
\text { 92 años }\end{array}$ & $-0,068$ & $-0,227-0,090$ & 0,396 \\
\hline $\begin{array}{l}\text { Tipo de fractura } \\
\text { extracapsular }\end{array}$ & $-0,027$ & $-0,197-0,843$ & 0,753 \\
\hline
\end{tabular}

*Valor $p<0,05$
Al realizar el estudio multivariable encontramos que el único factor protector de mortalidad al año es pertenecer al sistema de salud privado, sin asociarse el tiempo preoperatorio como encontramos en el estudio univariado. Eso puede explicarse debido a que, al utilizar el tiempo preoperatorio como variable categórica, la cantidad de pacientes encontrados en el grupo del SSPr que fueron operados después de las $72 \mathrm{hrs}$ fue muy pequeño, lo que no permite sacar conclusiones. Cabe destacar que el pertenecer a uno u otro sistema de salud, involucra múltiples factores asociados que pueden influir en la mortalidad de manera independiente pero que no fueron evaluados en este trabajo.

Este estudio corresponde a un estudio epidemiológico retrospectivo observacional por lo que cuenta con las limitaciones propias del diseño. No realizamos estudios de comorbilidades, como fue mencionado previamente, siendo esta nuestra mayor limitación para poder realizar conclusiones mayores. Sería interesante realizar estudios prospectivos y evaluar diferentes factores asociados a mortalidad que no fueron incluidos en el presente trabajo.

\section{Conclusión}

La mortalidad al año encontrada en el presente estudio es de $30,2 \%$ siendo significativamente mayor en pacientes pertenecientes al sistema público de salud. Encontramos asociación estadística entre el mayor tiempo preoperatorio y el aumento de la mortalidad al año. Es necesario estudiar el efecto en la mortalidad de otros factores que no fueron evaluados en el presente trabajo como es la presencia de comorbilidad. Este estudio aporta a la contingencia nacional en describir cómo se comporta nuestra población nonagenaria operada por fractura de cadera tanto en el ámbito privado como en el público. 


\section{Conflicto de Intereses}

Los autores declaran no tener conflictos de interés.

\section{Bibliografía}

1 http://www.who.int [Internet]. World Health Organization, 2016 [Actualizado 06 jun 2016, citado 19 may 2017] Disponible en: http://www.who.int/ageing/about/facts/es/

2 http://www.who.int [Internet]. World Health Organization, 2016 [Actualizado 06 jun 2016, citado 16 may 2017] Disponible en http://apps.who.int/gho/data/view.main.WOMENLEXv

3 http://www.deis.cl [Internet]. Departamento de Estadísticas e Información de Salud, 2017[Citado 16 may 2017]. Disponible en http://www.deis.cl/estadisticas-poblacion/

4 Hausdorff JM, Rios DA, Edelberg HK. Gait variability and fall risk in community-living older adults: a 1-year prospective study. Arch Phys Med Rehabil 2001;82(08):1050-1056

5 Shah MR, Aharonoff GB, Wolinsky P, Zuckerman JD, Koval KJ. Outcome after hip fracture in individuals ninety years of age and older. J Orthop Trauma 2001;15(01):34-39

6 Barangan JD. Factors that influence recovery from hip fracture during hospitalization. Orthop Nurs 1990;9(05):19-30

7 Gordon PC. The probability of death following a fracture of the hip. Can Med Assoc J 1971;105(01):47-51, passim

8 Intiso D, Di Rienzo F, Grimaldi G, et al. Survival and functional outcome in patients 90 years of age or older after hip fracture. Age Ageing 2009;38(05):619-622

9 Jette AM, Harris BA, Cleary PD, Campion EW. Functional recovery after hip fracture. Arch Phys Med Rehabil 1987;68(10):735-740

10 Cobey JC, Cobey JH, Conant L, Weil UH, Greenwald WF, Southwick WO. Indicators of recovery from fractures of the hip. Clin Orthop Relat Res 1976;(117):258-262

11 Mossey JM, Mutran E, Knott K, Craik R. Determinants of recovery 12 months after hip fracture: the importance of psychosocial factors. Am J Public Health 1989;79(03):279-286

12 Kadowaki M, Kono M, Nishiguchi K, Kakimaru H, Uchio Y. Mortality in patients with hip fracture aged over 90 years: a report from a progressively aging island. Arch Gerontol Geriatr 2012;54(02):e113-e117
13 Holvik K, Ranhoff AH, Martinsen MI, Solheim LF. Predictors of mortality in older hip fracture inpatients admitted to an orthogeriatric unit in oslo, norway. J Aging Health 2010;22(08): 1114-1131

14 Pedrera Zamorano JD, Bote Mohedano JL, Lavado García JM, Rodríguez Domínguez MT, Hernández Díaz ER, Rico Lenza H. Incidencia y prevalencia de las fracturas de cadera en la provincia de Cáceres y su tendencia evolutiva. Rev Clin Esp 2004;204(09):448-451

15 Gjertsen J. Surgical treatment of hip fractures in Norway [master's thesis]. Bergen (BE): University of Bergen; 2009:67

16 Luna L, Romo I, Fernández C, Cima M, García R, Hernández D. Epidemiología de las fracturas de la extremidad proximal del fémur en Asturias. Rev Esp Cir Osteoart 1993;28:261-266

17 Simunovic N, Devereaux PJ, Sprague S, et al. Effect of early surgery after hip fracture on mortality and complications: systematic review and meta-analysis. CMAJ 2010;182(15):1609-1616

18 Lin W-T, Chao C-M, Liu H-C, Li Y-J, Lee W-J, Lai C-C. Short-term outcomes of hip fractures in patients aged 90 years old and over receiving surgical intervention. PLoS One 2015;10(05):e0125496. Doi: $10.1371 /$ journal.pone.0125496

19 de Leur K, Vroemen JP, Vos DI, Elmans L, van der Laan L. Outcome after osteosynthesis of hip fractures in nonagenarians. Clin Interv Aging 2014;9:41-49

20 González-Zabaleta J, Pita-Fernandez S, Seoane-Pillado T, LópezCalviño B, Gonzalez-Zabaleta JL. Comorbidity as a predictor of mortality and mobility after hip fracture. Geriatr Gerontol Int 2016;16(05):561-569

21 Takamine B, Morii T, Watanabe H, Tajima T, Satomi K. Short-term postoperative mortality events in patients over 80 years of age with hip fracture: analysis at a single institution with limited medical resources. J Orthop Sci 2010;15(04):437-442

22 Holt G, Macdonald D, Fraser M, Reece AT. Outcome after surgery for fracture of the hip in patients aged over 95 years. J Bone Joint Surg Br 2006;88(08):1060-1064

23 Scottish Hip Fracture Audit - Report 2002. Information and Statistics Division, Edinburgh

24 Tol MC, van den Bekerom MP, Sierevelt IN, HilverdinkEF, Raaymakers EL, Goslings JC. Hemiarthroplasty or total hip arthroplasty for the treatment of a displaced intracapsular fracture in active elderly patients: 12-year follow-up of randomised trial. Bone Joint J 2017; 99-B(02):250-254 